\title{
Appendiceal neuroendocrine tumors in single center literature review and a case series
}

\begin{abstract}
The appendix is one of the most common single site for carcinoid tumor.1 Histopathologically, appendiceal neuroendocrine tumors (aNETs) is mostly comprised of enterochromaffin (EC) cell type and derives from a subepithelial cell population, which is different from neuroendocrine tumor in other sites. Although rare, and usually detected incidentally in appendectomy, it is considered the most common type of appendiceal primary malignant lesion, and is found in $0.3 \%-0.9 \%$ of patients undergoing appendicectomy. 2 This tumor rarely presents with metastases. In surgical practice most surgeons encounter only one or two such lesions during their career. Therefore, it is important to define correct management of such a rare tumor. We would like to report a series of 2 aNETs found during appendicectomy in a single centre and discuss about management strategies.
\end{abstract}

Volume 6 Issue 5 - 2018

Sarmukh Singh,' Kerwin Teoh,' Ramesh
Thangaratnam,' Chew Loon Guan,' Zaidi
Zakaria'
'Department of Surgery, Hospital Serdang, Selangor, Malaysia
'Department of Surgery, University Sains Malaysia, Malaysia

Correspondence: Sarmukh Singh, Department of Surgery, Hospital Serdang, Selangor, Malaysia, Tel 0179739329, Email sarmukh_rao@yahoo.com

Received: March 23, 2018| Published: September 27, 2018

Keywords: appendiceal neuroendocrine tumors, carcinoid tumor, appendicectomy, hemicolectomy, diarrhea, iliac fossa, retrocecal appendicitis, carcinoids, karzinoide, caecal, synaptophysin, chromogranin

Abbreviations: aNETs, appendiceal neuroendocrine tumors; HPE, histopathology examination; EC, enterochromaffin; SSAs, somatostatin analogues; RFA, radiofrequency ablation; aNENs, appendiceal neuroendocrine neoplasms; MAI, mesoappendiceal fat invasion; MRI, magnetic resonance imaging; SRI, somatostatin receptor imaging; US, ultrasound scan

\section{Case report}

Case 1: A 13-year-old Indian boy presented with right iliac fossa pain, vomiting, diarrhea and fever for the past 4 days. On further history taking, he also complained of weight loss about $7 \mathrm{~kg}$ in 1 month. Otherwise, there was no constitutional symptom. Patient has a strong family history of malignancy. Patient's mother was diagnosed breast cancer and patient's grandmother was diagnosed lingual cancer. Initial diagnosis was acute appendicitis. We proceeded with emergency open appendicectomy and intraoperative finding was inflamed retrocecal appendicitis, Tip of appendix was thickened and adhered deeply to the posterior peritoneum, otherwise caecal base was healthy and no Meckel's diverticulum found. Histopathology examination (HPE) result showed carcinoid tumor with tumor size $0.5 \mathrm{~cm}$ and base was free. Immunohistochemistry staining for Synaptophysin: Positive, Chromogranin: Positive and CD56: Positive. Urine 5-HIAA result was normal. CT Thorax, Abdomen and Pelvis showed no evidence of distant metastasis. We plan to follow up this patient every 6 monthly clinically.

Case 2: A 24-year-old Malay gentleman presented with right iliac fossa pain, diarrhea and fever for one day duration. Abdominal examination demonstrated tenderness over the right iliac fossa. Abdominal X-ray shows only fecal loaded over the ascending colon. Blood parameters were normal limits. Our initial diagnosis was acute appendicitis. We proceeded with emergency open appendicectomy. Intraoperative finding was perforated tip of appendix with $10 \mathrm{cc}$ pus but base was healthy. Patient was discharge home well at post operative day three. Patient was reviewed back in clinic in 1 month and the HPE result showed carcinoid tumor with tumor size $0.5 \mathrm{~cm}$, base and body is free. Immunohistochemistry staining for Synaptophysin: Positive, Chromogranin: Positive and CD56: Positive. Urine 5-HIAA and result was normal. CT Thorax, Abdomen and Pelvis showed no evidence of distant metastasis. We plan to follow up this patient every 6 monthly clinically.

\section{Discussion}

Oberndofer first described these tumors and used the term carcinoid (or "karzinoide") in 1907.In 1963 Williams and Sandler classified carcinoids according to embryogenetic aspects. The prevalence of aNETs is $0.3-0.9 \%$ in patients undergoing appendectomy, with the rated at 8.4 per 100000 population per year. This means that the diagnosis will be made in one in 100-300 operations. The most frequent 'carcinoids' (NETs) is neoplasms of the appendix, which is $32-57 \%$ of all appendiceal tumours. Gastrointestinal tract NETs present predominantly in the small intestine $44.7 \%$, followed by rectum $19.6 \%$, then the appendix which is $16.7 \%$. ANETs usually present at younger age group, with a peak in the fourth decade. ANETs occur more frequently in female gender.

Histological features predictive of aggressive tumor behavior are size, histological subtype and mesoappendiceal involvement. ${ }^{3}$ Most of the tumors size is smaller than $1 \mathrm{~cm}$ in diameter in $60-76 \%$ of patients. In tumor size $1-2 \mathrm{~cm}$ in diameter is $4-27 \%$ and tumor size larger than $2 \mathrm{~cm}$ in diameter is $2-17 \%$. There are 4 histologic subtypes of aNETs which are goblet cell, composite cell, enterochromaffin cell and atypical cell. The risk of metastasis from tumors smaller than 1 $\mathrm{cm}$ is zero, while tumor size larger than $2 \mathrm{~cm}$ increase the risk of metastasis from $20 \%-85 \%{ }^{4}$

In our case series, all the tumors were less than $1 \mathrm{~cm}$ and localized at the tip of appendix with no evidence of regional or distant metastases. ANETs usually metastasize to regional lymph nodes rather than to the liver. ${ }^{5}$ The aNETs distribution is heterogeneous, in which the tip of the appendix is the most frequent location $60-70 \%$ followed by in the body $5-21 \%$ and then the base of appendix $7-10 \%$ (Table 1 ). 
We would like to discuss about management approach based on the HPE characteristics for prognostic index aNETs. The size of the tumor and histological patterns are the most important factors that contribute to the selection of the surgical treatment. For aNETs less than $1 \mathrm{~cm}$ in diameter size, it is unlikely to have metastasized. Therefore, a simple appendectomy with clear margin would suffice. However if the aNETs measures more than $1 \mathrm{~cm}$ and less than $2 \mathrm{~cm}$ in diameter size, with no muscular invasion or lymph node involvement a simple appendectomy with surgical margins free of tumor. However, Goede $^{2}$ and Moertel $^{4}$ suggested in aNETs greater than $2 \mathrm{~cm}$ in diameter size with lymph node involvement and high-grade malignant carcinoids (including those with cellular pleomorphism and a high mitotic index, more than 2 mitoses/10 HPF), extension of the tumor into the mesoappendix, subserosal lymphatic invasion, tumor location near the base of the appendix or close to the cecum with potential involvement of its wall, and tumor-positive resection margins then this should be managed with right hemicolectomy. ${ }^{2,6}$ Meanwhile, in elderly age group and in high risk patients a simple appendectomy may be adequate for surgical resection of tumors larger than $2 \mathrm{~cm}$ in diameter in size (Table 2). ${ }^{4}$

Table 2 Show the summary of treatment based on the size of the aNETs
Table I Classifications and staging of aNETs according to the TNM system

\begin{tabular}{llll}
\cline { 2 - 4 } Stage & T & N & M \\
\cline { 2 - 4 } I & TI & N0 & M0 \\
& TI & N I & M0 \\
III & T2 & N0 & M0 \\
& T2 & N I & M0 \\
IV & T3 & Any N & M0 \\
& & Any N & MI \\
\hline
\end{tabular}

TI:Tumor $<2 \mathrm{~cm}$

T2: Tumor $\geq 2 \mathrm{~cm}$ but $<3 \mathrm{~cm}$

T3:Tumor $\geq 3 \mathrm{~cm}$

No: No lymph node metastases

$\mathrm{NI}$ : Regional lymph node metastases

M0: No metastases

MI: Distant metastases

\begin{tabular}{|c|c|c|c|c|c|}
\hline Tumor size & $\begin{array}{l}\text { Risk for } \\
\text { lymphatic } \\
\text { spread }\end{array}$ & Further characterization & $\begin{array}{l}\text { Predictive } \\
\text { validity for } \\
\text { malignancy }\end{array}$ & $\begin{array}{l}\text { Type of evidence } \\
\text { source }\end{array}$ & $\begin{array}{l}\text { Treatment } \\
\text { recommendations }\end{array}$ \\
\hline \multirow[t]{3}{*}{$<\mathrm{I} \mathrm{cm}$} & 0 & None (positive margins) & Accepted & $\begin{array}{l}\text { Retrospective series } \\
\text { (Conclusive) }\end{array}$ & Appendectomy \\
\hline & & Serosal invasion & $\begin{array}{l}\text { Not predictive } \\
\text { (accepted) }\end{array}$ & Retrospective series & \\
\hline & & Mesoappendiceal invasion & Controversial & Retrospective series & \\
\hline \multirow[t]{3}{*}{$\mathrm{I}-2 \mathrm{~cm}$} & & Vascular invasion & Controversial & Retrospective series & $\begin{array}{l}\text { Individual risk evaluation: } \\
\text { High risk (elderly) patient: }\end{array}$ \\
\hline & $0-1 \%$ & $\begin{array}{l}\text { Mitotic activity ( }>2 \text { cells } / \mathrm{mm}^{2} \text { ) } \\
\text { Proliferation markers (i.e. } \mathrm{Ki} 67 \text { ) }\end{array}$ & Not proven & $\begin{array}{l}\text { Common sense } \\
\text { evidence' (data from } \\
\text { different NETs applied } \\
\text { to appendix) }\end{array}$ & $\begin{array}{l}\text { Appendectomy 'High } \\
\text { risk' tumor, low risk } \\
\text { (younger) patient: Right } \\
\text { hemicolectomy }\end{array}$ \\
\hline & & $\begin{array}{l}\text { Localization at base of appendix } \\
\text { Positive margins }\end{array}$ & Accepted & $\begin{array}{l}\text { 'Common sense } \\
\text { evidence' }\end{array}$ & \\
\hline$>2 \mathrm{~cm}$ & $30 \%$ & None & Accepted & $\begin{array}{l}\text { Retrospective series } \\
\text { (Conclusive) }\end{array}$ & Right hemicolectomy \\
\hline $\begin{array}{l}\text { Any size goblet- } \\
\text { cell tumor }\end{array}$ & $10-20 \%$ & None & Accepted & $\begin{array}{l}\text { Retrospective series } \\
\text { (Conclusive) }\end{array}$ & Right hemicolectomy \\
\hline
\end{tabular}

In metastatic tumors and carcinoid syndrome the aNETs with lymph node metastases and vascular invasion is as high as $30 \%$. Only $1 \%$ for tumors with appendiceal mesentery invasion. Right hemicolectomy should be considered in patients with more extensive (stage III and VI) disease. ${ }^{7}$ However, Alexandraki ${ }^{7}$ suggested to use systemic therapies such as somatostatin analogues (SSAs) / octreotide in this group of patients as it can prolonged progression and improve symptoms associated with carcinoid syndrome with a clinical and biochemical response rate of up to $60 \%{ }^{8} \mathrm{Pape}^{6}$ suggested patients with progressive disease beside treating with SSAs, further locoregional therapies such as radiofrequency ablation (RFA) could be used in patients with hepatic metastases. Chemotherapy is not use in metastatic tumor, is because of its poor response rate which is less than $40 \% .^{9}$ The 5 -year survival rate of patients with local disease is reported to be $92 \%$, those with regional metastases to be $81 \%$ and those with distant metastases was $31 \%$ respectively (Figure 1). ${ }^{10}$ 


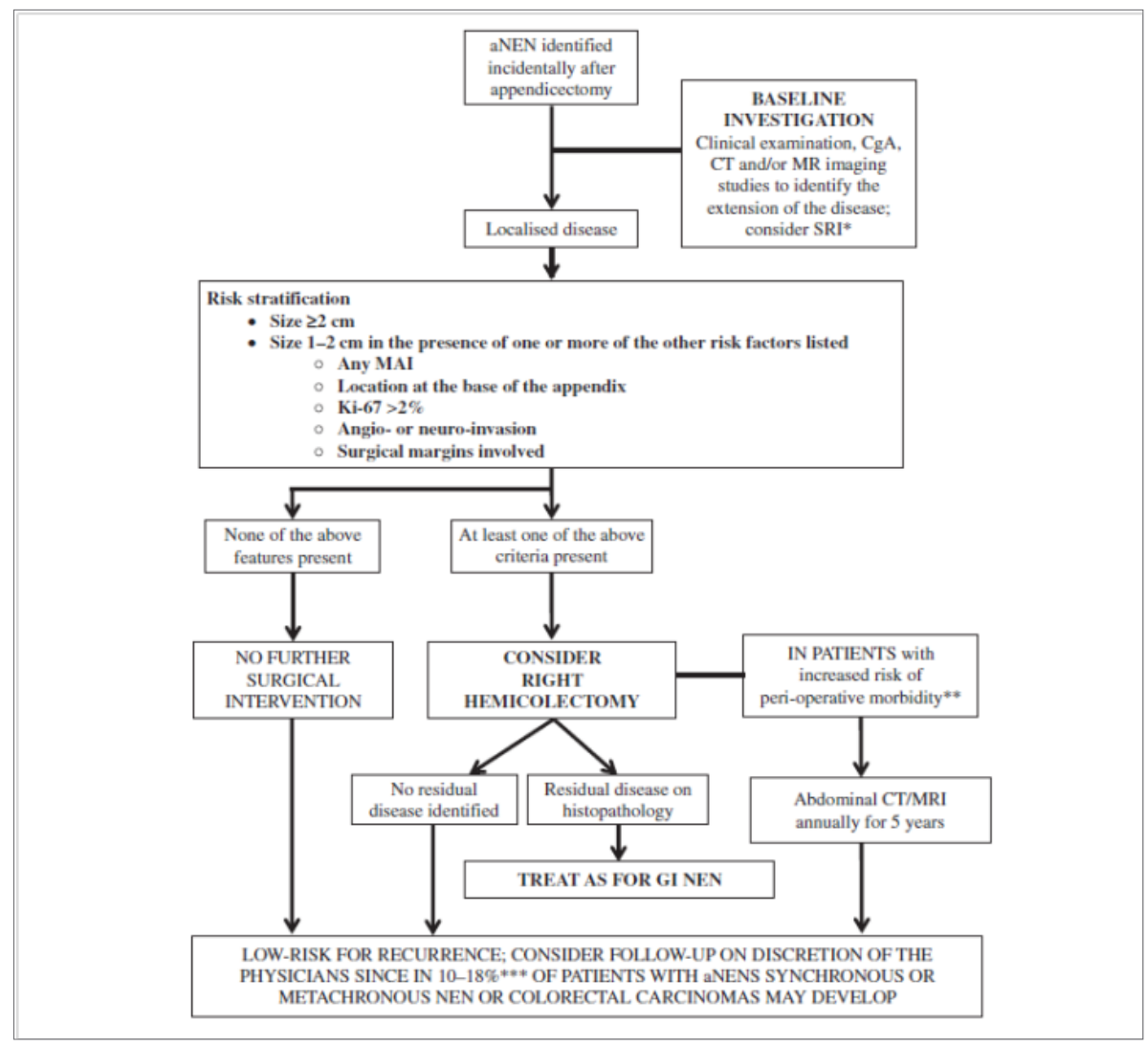

Figure I Algorithm for appendiceal NETs therapeutic and follow-up in incidentally found after appendicectomy for acute appendicitis, aNENs, appendiceal neuroendocrine neoplasms; MAI, mesoappendiceal fat invasion; MRI, magnetic resonance imaging; NEN, neuroendocrine neoplasm; SRI, somatostatin receptor imaging; US, ultrasound scan.

\section{Conclusion}

ANETs commonly presents as acute appendicitis. In most cases, it is found incidentally during appendectomies and its diagnosis is rarely suspected before histological examination. Although, aNETs has an excellent overall prognosis, consideration should be given to follow up this group of patients. At the time of diagnosis, we would recommend clinical examination and laboratory investigations with CgA and CT imaging. ${ }^{11}$ In our opinion, 6 monthly follow up is indicated in the low-risk tumors which is less than $1 \mathrm{~cm}$ in the largest diameter with no mesoappendiceal fat invasion presence and the location at the tip or intermediate part of the appendix with low Ki-67 and no other extension. However, if there is evidence of residual disease following Right hemicolectomy, a yearly CT abdominal scanning together with blood $\mathrm{CgA}$ is recommended as safe strategy. ${ }^{12}$

\section{Acknowledgements}

None.

\section{Conflict of interest}

Author declares that there is no conflict of interest.

\section{References}

1. Modlin IM, Lye KD, Kidd M. A 5-decade analysis of 13,715 carcinoid tumors. Cancer. 2003;97(4):934-959.

2. Goede AC, Caplin ME, Winslet MC. Carcinoid tumour of the appendix. $\mathrm{Br}$ J Surg. 2003;90(11):1317-1322.
3. Connor SJ, Hanna GB, Frizelle FA. Appendiceal tumors: retrospective clinicopathologic analysis of appendiceal tumors from 7,970 appendectomies. Dis Colon Rectum. 1998;41(1):75-80.

4. Moertel CG, Weiland LH, Nagorney DM, et al. Carcinoid tumor of the appendix: treatment and prognosis. $N$ Engl J Med. 1987;317(21):16991701.

5. Alexandraki KI, Griniatsos J, Bramis KI et al. Clinical value of right hemicolectomy for appendiceal carcinoids using pathologic criteria. $J$ Endocrinol Invest. 2011;34(4):255-259.

6. Safioleas MC, Moulakakis KG, Kontzoglou K, et al. Carcinoid tumors of the appendix. Prognostic factors and evaluation of indications for right hemicolectomy. Hepatogastroenterology. 2005;52(61):123-127.

7. Pape UF, Perren A, Niederle B, et al. ENETS Consensus Guidelines for the management of patients with neuroendocrine neoplasms from the jejuno-ileum and the appendix including goblet cell carcinomas. Neuroendocrinology. 2012;95(2):135-156.

8. Anderson JR, Wilson BG. Carcinoid tumours of the appendix. Br J Surg. 1985;72(7):545-546.

9. Thompson GB, van Heerden JA, Martin JK, et al. Carcinoid tumors of the gastrointestinal tract: presentation, management, and prognosis. Surgery. 1985;98(6):1054-1063.

10. Sweeney JF, Rosemurgy AS. Carcinoid Tumors of the Gut. Cancer Control. 1997;4(1):18-24.

11. Kvols LK. Metastatic carcinoid tumors and the carcinoid syndrome. A selective review of chemotherapy and hormonal therapy. Am J Med. 1986;81(6B):49-55. 
12. Moertel CG, Rubin J, Kvols LK. Therapy of metastatic carcinoid tumor and the malignant carcinoid syndrome with recombinant leukocyte A interferon. J Clin Oncol. 1989;7(7):865-868. 